\title{
Synthetic modelling of the light-travel time effect of circumbinary planets
}

\author{
Tobias C. Hinse and Jae Woo Lee \\ Korea Astronomy \& Space Science Institute, 305-348 Daejeon, Republic of Korea \\ email: tchinse@gmail.com
}

\begin{abstract}
In recent years several multi-body, circumbinary planets have been proposed to orbit short-period eclipsing binaries. In light of the recent discoveries based on the Kepler data, the existence of such systems seems plausible. However, performing a detailed dynamical analysis reveals that the majority of the proposed planetary systems follow highly unstable orbits. In order to solve the origin of this problem, we have started to model synthetic light-travel time signals of stable planetary systems. In particular, we aim to study the response of the model in various circumstances (e.g red/white noise level, various sampling frequencies, in-homogeneous data sets, baseline dependency.) This work will significantly increase the confidence with which model work is carried out for future systems and help towards an understaning when models break down (e.g resulting in unstable systems).
\end{abstract}

Keywords. binaries: eclipsing, planetary systems, methods: data analysis, formation: planetary systems

\section{Introduction}

Lately, several multi-body systems have been proposed (Lee et al. 2009, Qian et al. 2011, Almeida et al. 2013) to orbit a short-period, eclipsing binary. The light-travel time effect can invoked to detect additional bodies. By measuring the times of eclipses, it is possible to detect small timing variations. At some times the binary system will be closer to Earth, and at other times it will be positioned farther away. These circumbinary companions are of planetary nature with mass between 3 and $10 M_{J u p}$. In light of the recent discoveries (e.g. Kepler-16, Kepler-47) from the Kepler space telescope (e.g. Doyle et al. 2011, Orosz et al. 2012, Welsh et al. 2012) such systems seem plausible. For Kepler47 the two planets orbit the binary on stable orbits with lifetimes of over 100 million years.

However, those multi-body circumbinary companions proposed from invoking the lighttravel time effect are often following orbits that are short-lived in comparison to the systems overall age (Horner et al. 2011, Goździewski et al. 2012, Hinse et al. 2012). This finding raises some doubts about the confidence of their existence. Although the possibility exists that the detected timing variations are caused by additional companions, the currently proposed systems must, at least, exhibit a different orbital architecture to ensure long-term orbital stability. Usually, stable planetary systems are characterised by well-separated, non-crossing orbits with orbital eccentricities close to zero. However, for most of the proposed systems the orbits of the two planets are crossing implying mutual encounters resulting in catastrophic break-up events.

\section{Synthetic modelling of light-travel time signal}

This paper marks the beginning of a series of research efforts addressing the modelling of synthetic one- and two-companion light-travel time signals. We generate synthetic 

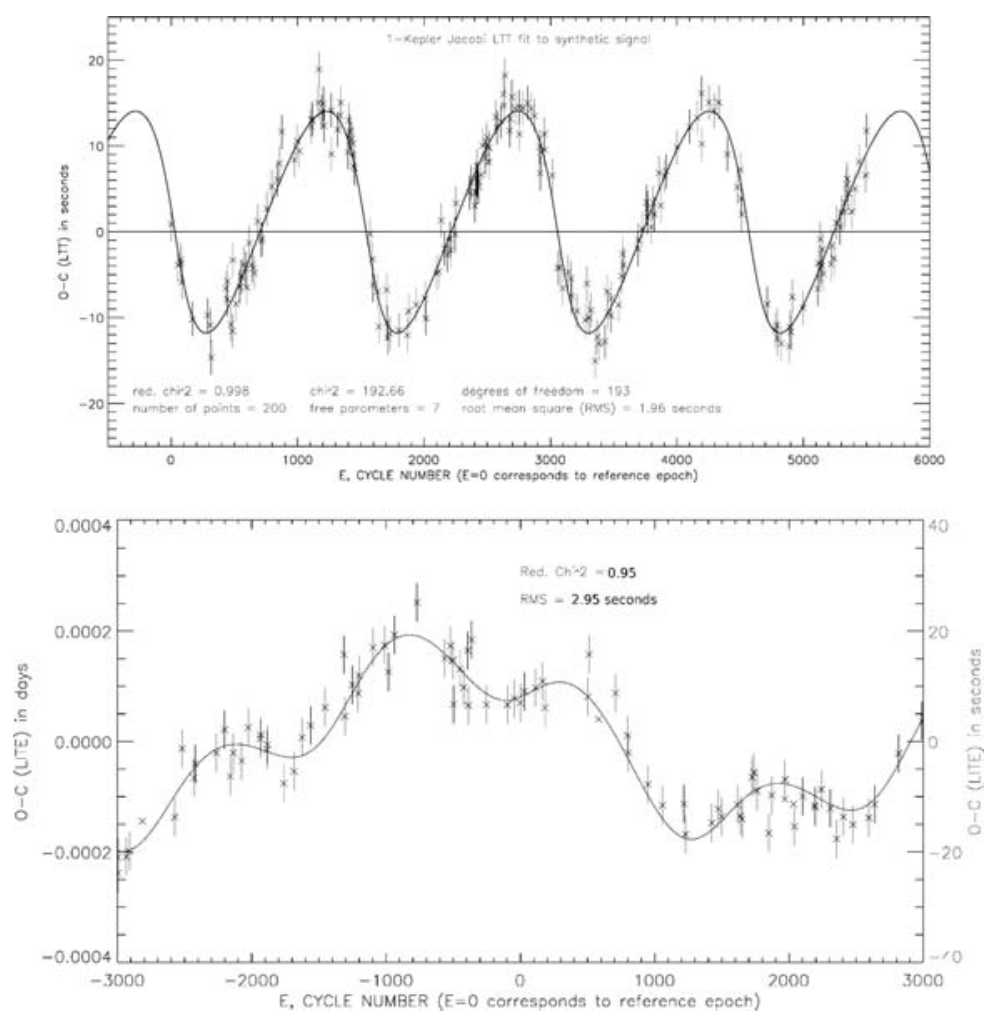

Figure 1. Synthetic modelling of light-travel time signals from a single (top panel) and twocompanion (bottom panel) system. Recently, Conroy et al. (2013) announced the existence of several one-companion systems orbiting a short-period binary.

signals of stable circumbinary planetary systems containig one or more companions. We have generated single and two-companion signals (Fig. 1) with Gaussian noise added.

We base our model on the Jacobian formalism as presented in Goździewski et al. (2012). The original signals were sucessfully recovered even though the mutual gravitational interactions have not been taken into account in the modelling work of the two-companion case. In the future we aim to study the dependence of parameter errors on the timing precision and examine the effect of considering various baselines on the final model parameters.

Acknowledgment: This research is supported by KASI grant 2013-9-400-00 and financial support by the IAU.

\section{References}

Almeida, L. A., Jablonski, F., \& Rodrigues, C. V., 2013, ApJ, 766, 11

Conroy, K. E., et al. 2013, arXiv:1306.0512, submitted to AJ

Doyle, L. R., et al. 2011, Science, 333, 1602

Goździewski, K., et al. 2012, MNRAS, 425, 930

Hinse, T. C., et al. 2012, MNRAS, 420, 3609

Horner, J., Marshall, J. P., Wittenmyer, R. A., \& Tinney, C. G. 2011, MNRAS, 416, 11

Lee, J. W., et al. 2009, AJ, 137, 3181

Orosz, J. A., et al. 2012b, Science, 337, 1511

Qian, S.-B., et al. 2011, MNRAS, 414, 16

Welsh, W., et al. 2012, Nature, 481, 475 